\title{
Long-Term Follow-Up of Patient with Diabetic Macular Edema Receiving Fluocinolone Acetonide Intravitreal Implant
}

\author{
Thomas Bertelmann · Stephan Schulze
}

To view enhanced content go to www.ophthalmology-open.com

Received: December 2, 2014 / Published online: February 12, 2015

(C) The Author(s) 2015. This article is published with open access at Springerlink.com

\section{ABSTRACT}

Introduction: This case study aims to evaluate outcomes following fluocinolone acetonide [FAc $0.2 \mu \mathrm{g} /$ day; ILUVIEN ${ }^{\circledR}$ (Alimera Sciences Limited, Aldershot, UK)] implant in a patient with diabetic macular edema (DME) not responding to laser photocoagulation or antivascular endothelial growth factor (anti-VEGF) therapy and to compare FAc implant with antiVEGF therapy in the fellow eye.

Case Report: The author presents here a patient with DME for around 20 years in both eyes, who had undergone pan-retinal and focal photocoagulation, and anti-VEGF therapy in both eyes without resolution of DME. FAc implant in the left, and subsequently in the right eye, provided substantial improvements in edema and visual acuity.

Conclusion: In the current case, a benefit was demonstrated in the FAc implant-treated left

Electronic supplementary material The online version of this article (doi:10.1007/s40123-015-0028-0) contains supplementary material, which is available to authorized users.

T. Bertelmann $(\varangle) \cdot S$. Schulze

Department of Ophthalmology, Philipps University, Baldingerstraße, 35043 Marburg, Germany

e-mail: thomas.bertelmann@staff.uni-marburg.de eye at a time when the right eye was not responding to anti-VEGF injections. If a patient does not respond well to an anti-VEGF (i.e. firstline therapy) in one eye, the treating physician should consider switching the patient to a corticosteroid implant (such as FAc implant) in the fellow eye.

Keywords: Diabetic macular edema; Fluocinolone acetonide; ILUVIEN ${ }^{\circledR}$; Ocular coherence tomography; Visual acuity

\section{INTRODUCTION}

As a chronic, enduring condition, diabetic macular edema (DME) has a significant burden of low vision and blindness [1], is difficult to manage, and therapeutic agents providing sustained benefit are needed [2]. While DME was once solely treated with laser therapy, a large number of patients continued to lose vision, despite treatment $[3,4]$. Although antivascular endothelial growth factor (anti-VEGF) therapy has become the preferred treatment for the management of DME, it is not effective in all patients, as demonstrated in the RIDE (ClinicalTrials.gov NCT00473382) and RISE 
(ClinicalTrials.gov NCT00473330) clinical trials, where between $58 \%$ and $70 \%$ of patients gained a best corrected visual acuity (BCVA) in Early Treatment Diabetic Retinopathy Study (EDTRS) letter score of $\geq 10$ suggesting approximately one-third of patients were insufficiently responsive to anti-VEGF therapy [5].

Intravitreal steroids, particularly in sustainedrelease implants, can offer a different DME therapeutic strategy, by providing localized delivery of the corticosteroid to maximize its anti-inflammatory, angiostatic and antipermeability effects, as well as minimize risks of systemic toxicity [3]. Fluocinolone acetonide $\left[0.2 \mu \mathrm{g} /\right.$ day FAc implant; ILUVIEN ${ }^{\circledR}$ (Alimera Sciences Limited, Aldershot, UK)] is a nonbiodegradable intravitreal corticosteroid implant that is delivered using a 25-gauge injector $[6,7]$. One implant releases sustained, low-dose FAc $(0.2 \mu \mathrm{g} /$ day $)$ for up to 3 years [8].

Currently, no direct comparative data exist for anti-VEGF versus FAc implant therapy in DME unresponsive to other therapies. Here, the authors describe a patient case that provides both direct comparison between anti-VEGF and FAc implant in the fellow eye, and also insight into the relative efficacy of FAc implant over more than 1 year in DME not responding to anti-VEGF therapy. This case has been reported previously, but follow-up was limited to 12 weeks in one eye, without comparison to the anti-VEGF-treated fellow eye [9].

\section{CASE REPORT}

This case is from a 30-year-old patient with typeI diabetes who was diagnosed with DME around 20 years ago. The patient's glycated hemoglobin (HbA1c) was 9.5\% and he was receiving insulin therapy. The patient had previously undergone pan-retinal and focal photocoagulation in both eyes. Anti-VEGF therapy had also been administered to both eyes prior to FAc implant, with the right and left eye receiving 15 and 12 intravitreal ranibizumab injections, respectively, since 2011; injections were administered monthly wherever possible, although treatment interruptions did occur due to reimbursement issues and challenging patient compliance. This report was carried out following informed consent from the patient.

\section{Treatment of the Left Eye}

The patient received an FAc implant on August 16, 2013, at which time central macular thickness (CMT) was $642 \mu \mathrm{m}$. Following FAc implant, CMT decreased rapidly and markedly ( $-364 \mu \mathrm{m}$ at month 1$)$ decreasing to $278 \mu \mathrm{m}$ at its minimum; the edema resolved quickly following FAc implant, despite epiretinal membrane formation (Fig. 1a). CMT remained below $330 \mu \mathrm{m}$ up to month 3 and then increased slightly, measuring $442 \mu \mathrm{m}$ at month 9 and $372 \mu \mathrm{m}$ at month 13 , still remaining more than $250 \mu \mathrm{m}$ below baseline CMT (Figs. 1, 2).

Best corrected visual acuity also increased rapidly following FAc implant in the left eye (Fig. 3; Table 1) improving from 0.3 at baseline to 0.5 after 1 month. This change was sustained through to month 9, although at month 13 BCVA decreased slightly to 0.4 as a result of cataract formation, which was confirmed by the return of BCVA to 0.51 day after phacoemulsification. The macula at this point still remained dry (see Fig. 1f).

\section{Treatment of the Right Eye}

The right eye was treated with a further three injections of ranibizumab before an FAc implant was injected (Table 1), a total of 

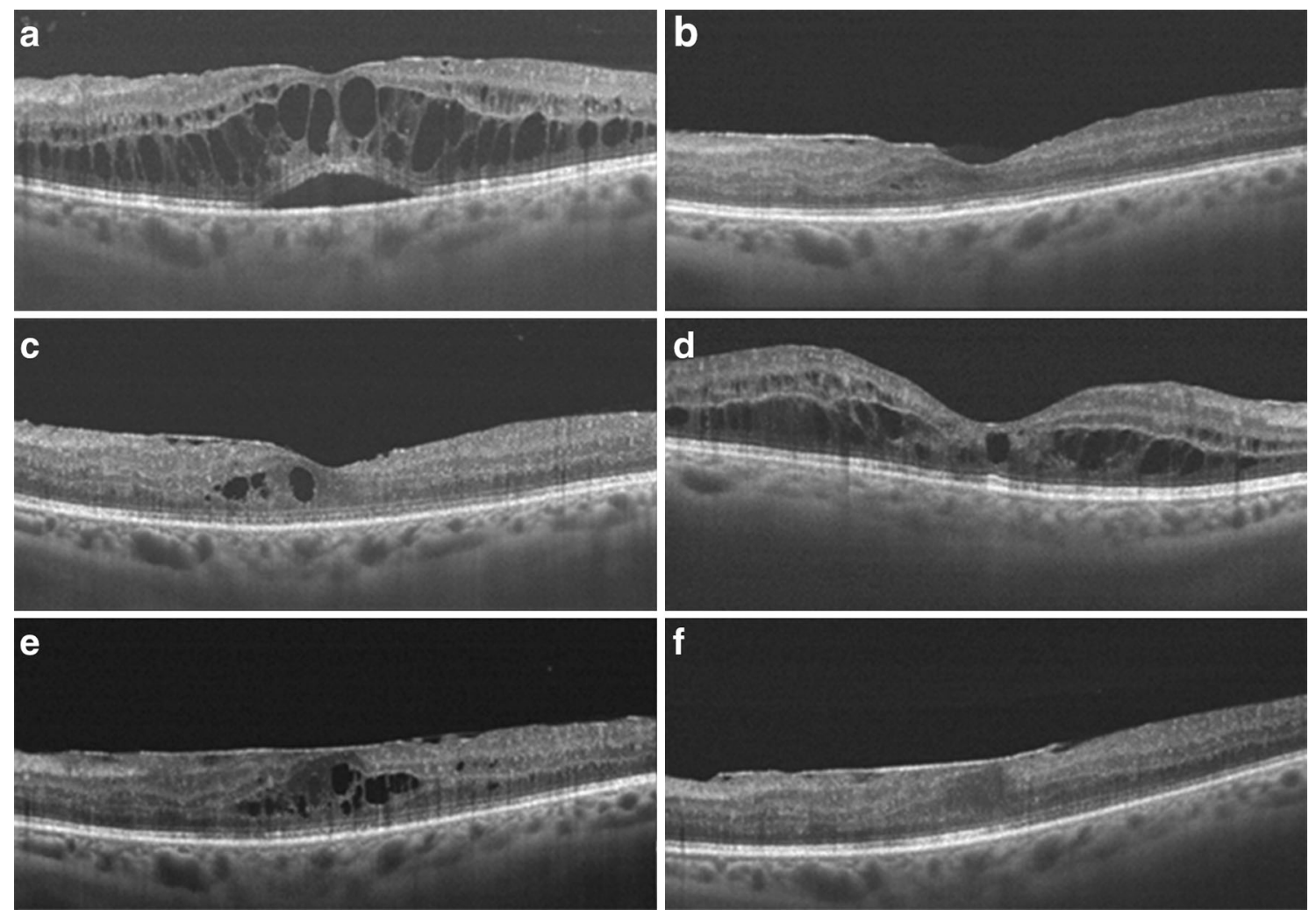

Fig. 1 Changes in macular thickness in the left eye before and after fluocinolone acetonide (FAc) implant. Optical coherence tomography (OCT) scans of left eye diabetic maculopathy in a patient with diabetic macular edema

(DME); a before treatment; b 1 month following FAc implant; c 3 months after FAc implant; d 7 months postFAc implant injection; e 9 months following treatment; and $\mathbf{f} 13$ months after FAc implant injection

18 anti-VEGF injections. CMT was $654 \mu \mathrm{m}$ prior to ranibizumab injections, which decreased thickness to $473 \mu \mathrm{m}$. However, 1 month after FAc implant, CMT decreased from 473 to $263 \mu \mathrm{m} \quad(-210 \mu \mathrm{m}) \quad$ increasing slightly to $334 \mu \mathrm{m}$ after 6 months (Table 1; Figs. 3, 4).

In the 7 months prior to FAc implant, BCVA remained $\leq 0.5$ in the right eye (Table 1 ). At 1 and 6 months after FAc implant, BCVA had increased to 0.6 and 0.7 , respectively.

\section{Adverse Events}

An increase in intraocular pressure (IOP) was seen in the left eye of the current patient, rising from $20 \mathrm{mmHg}$ at month 3 to $32 \mathrm{mmHg}$, at month 7 . This change was managed with $10 \mathrm{mg}$

brinzolamide/5 $\mathrm{mg}$ timolol eye drops which reduced IOP to $16 \mathrm{mmHg}$. At 13 months, left and right eye IOP (6 months after FAc implant had been administered) was 21 and $18 \mathrm{mmHg}$, respectively.

No further adverse events have been reported to date, at the time this case study was accepted for publication (January 19, 2015).

\section{DISCUSSION}

FAc implant $0.2 \mu \mathrm{g} /$ day is a second-line therapy indicated for the treatment of vision impairment associated with chronic diabetic macular edema, considered insufficiently responsive to available therapies $[8,10]$. In the present case, the patient had not responded 


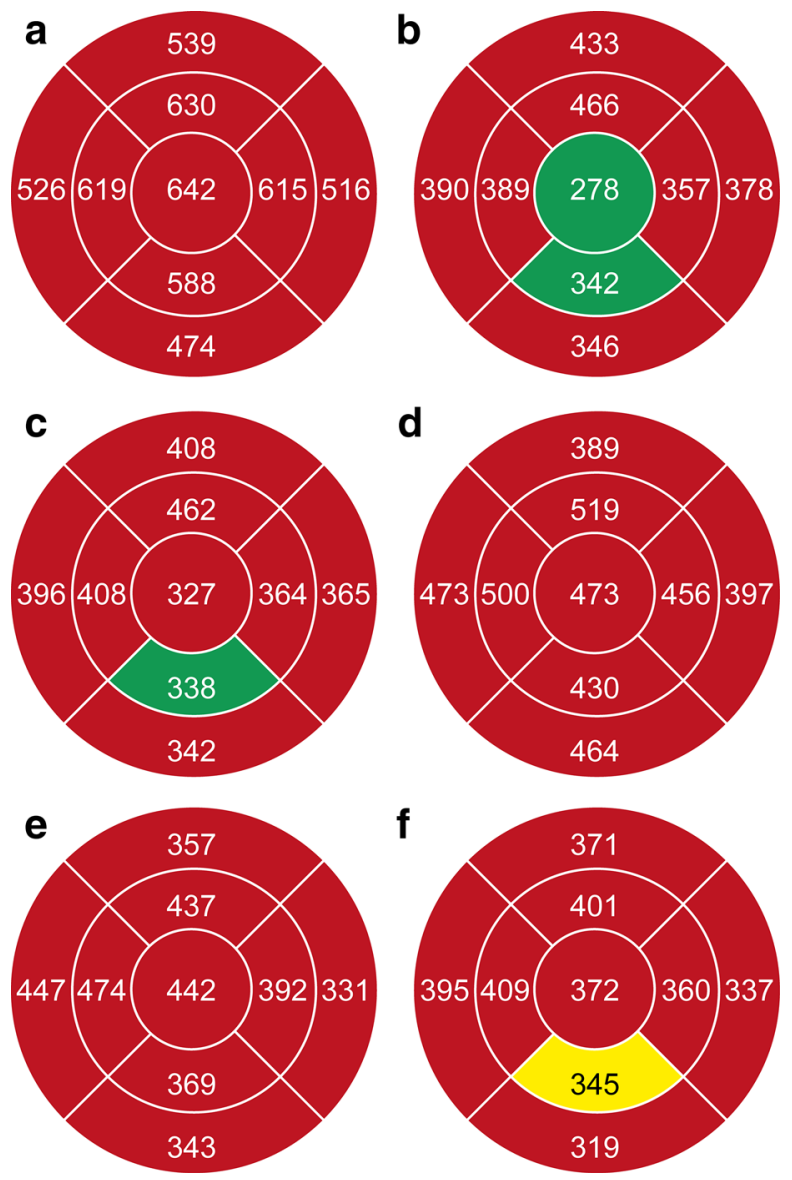

Fig. 2 Changes in retinal thickness $(\mu \mathrm{m})$ in the left eye before and after fluocinolone acetonide (FAc) implant. Optical coherence tomography (OCT) retinal thickness maps of left eye diabetic maculopathy in a patient with diabetic macular edema (DME); a before treatment; b 1 month following FAc implant; c 3 months after FAc implant; d 7 months post-FAc implant injection; e 9 months following treatment; and f 13 months after FAc implant injection. Color indications: Green (displays retinal thickness within normal limits), Yellow (retinal thickness marginally outside normal limits), Red (retinal thickness substantially outside normal limits)

sufficiently to prior treatments, which included both laser and anti-VEGF therapy. This is critically important in the chronic setting, since intraretinal fluid accumulation resulting in significant visual acuity reduction may be reversible with continued anti-VEGF therapy, but the prolonged edema can cause irreversible damage resulting in permanent visual loss [1].
The rapid resolution in both edema and BCVA after FAc implant seen in this case (as early as 1 month post-implant) was also seen in the FAME studies (ClinicalTrials.gov NCT00344968) [2] where, in the chronic DME group, central foveal thickness decreased by week 6 and was sustained through to 36 months [8]; however, subjects needed cataract surgery more frequently following $0.2 \mu \mathrm{g} /$ day $\mathrm{FAc}$ implant than sham injection [2].

Two new insights relating to the indication for FAc implant and its position in the treatment algorithm are suggested by this case. The first relates to the indication statement that FAc implant is 'for the treatment of vision impairment associated with chronic DME, considered insufficiently responsive to available therapies' [8]. The current case provides a direct comparison between the response to FAc implant, in the left eye, and anti-VEGF, in the right eye (Table 1). During the first 7 months following FAc implant, the left eye showed rapid and marked improvements in BCVA and CMT, which were sustained. In contrast, BCVA did not improve in the right eye, the change in CMT was roughly half that of the left eye, and the macula in the right eye remained swollen and wet (Fig. 1), which could suggest that in this chronic DME case, anti-VEGF was less effective than FAc implant. This is further supported by the subsequent response of the right eye to FAc implant. A clear improvement in BCVA and CMT was observed up to 6 months after FAc implant that achieved similar absolute values to those in the left eye.

The second insight relates to the timing of therapies. Prior to FAc implant being administered, the patient had received multiple anti-VEGF injections. Although the left eye responded to FAc implant, anti-VEGF treatment was continued for the right eye, with 
a

\section{BCVA (left eye)}

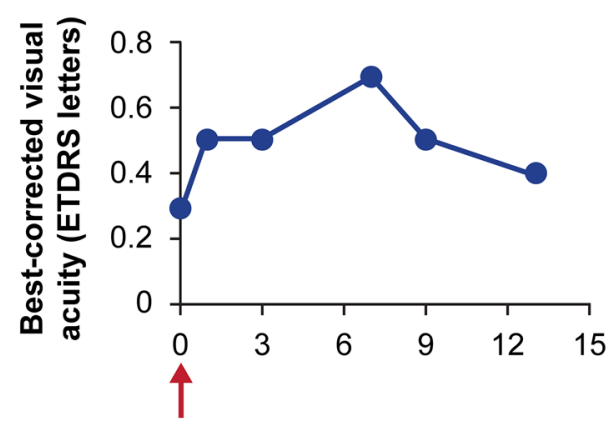

C

CMT (left eye)

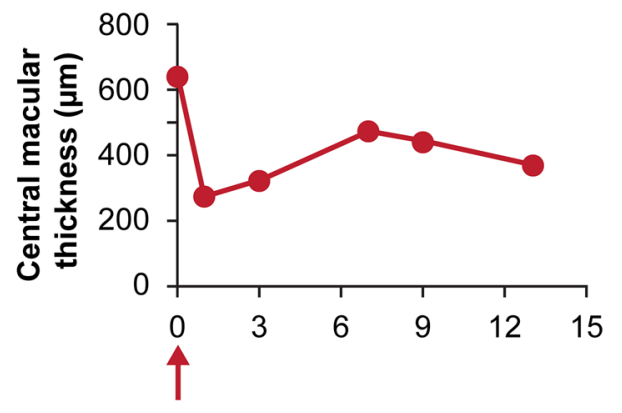

Time (months)

Fig. 3 Changes in best corrected visual acuity (BCVA) and central macular thickness (CMT) before and after fluocinolone acetonide (FAc) implant. BCVA in a left eye and $\mathbf{b}$ right eye, and CMT in $\mathbf{c}$ left eye and $\mathbf{d}$ right eye b

\section{BCVA (right eye)}

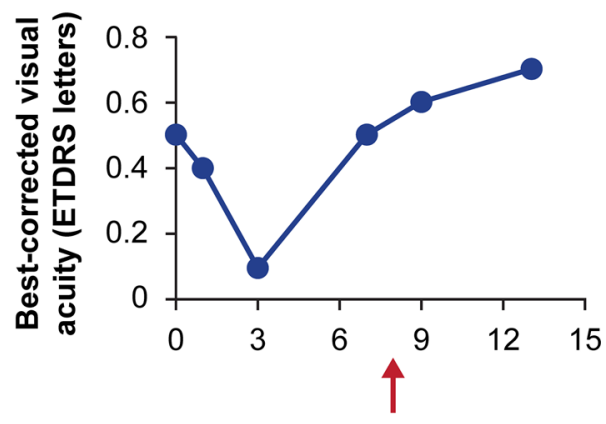

d

\section{CMT (right eye)}

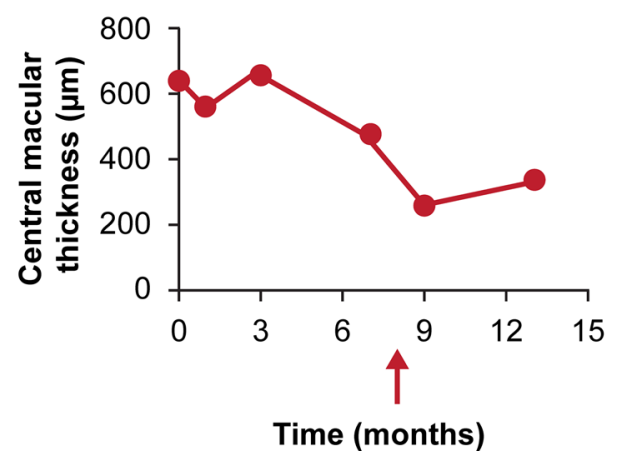

following FAc implant. Red arrow indicates when the FAc implant was first inserted. ETDRS Early treatment Diabetic Retinopathy study

Table 1 Changes in best corrected visual acuity (BCVA) and central macular thickness (CMT) before and after fluocinolone acetonide (FAc) implant

\begin{tabular}{|c|c|c|c|c|c|}
\hline \multicolumn{3}{|l|}{ Left eye } & \multicolumn{3}{|l|}{ Right eye } \\
\hline Time post-FAc implant & BCVA & $\overline{\mathrm{CMT}}(\boldsymbol{\mu \mathrm { m }})$ & Time post-FAc implant & BCVA & $\overline{\mathrm{CMT}}(\boldsymbol{\mu \mathrm { m }})$ \\
\hline Baseline & 0.3 & 642.0 & -7 months & 0.5 & 639.0 \\
\hline+1 month & 0.5 & 278.0 & -6 months & 0.4 & 568.0 \\
\hline+3 months & 0.5 & 327.0 & -4 months & 0.1 & 654.0 \\
\hline+7 months & 0.7 & 473.0 & -1 month (baseline) & $0.5^{\mathrm{a}}$ & 473.0 \\
\hline+9 months & 0.5 & 442.0 & +1 month & 0.6 & 263.0 \\
\hline+13 months & $0.4^{\mathrm{b}}$ & 372.0 & +6 months & 0.7 & 334.0 \\
\hline
\end{tabular}

a Three further injections of ranibizumab (i.e., a total of 18 injections) had been administered prior to this time point

b At 13 months, BCVA in the left eye was 0.4, with the decrease being thought to relate to the formation of a cataract. Phacoemulsification was conducted at this stage and on the first post-operative day, visual acuity had increased to 0.5 . The macula at this point was still dry 


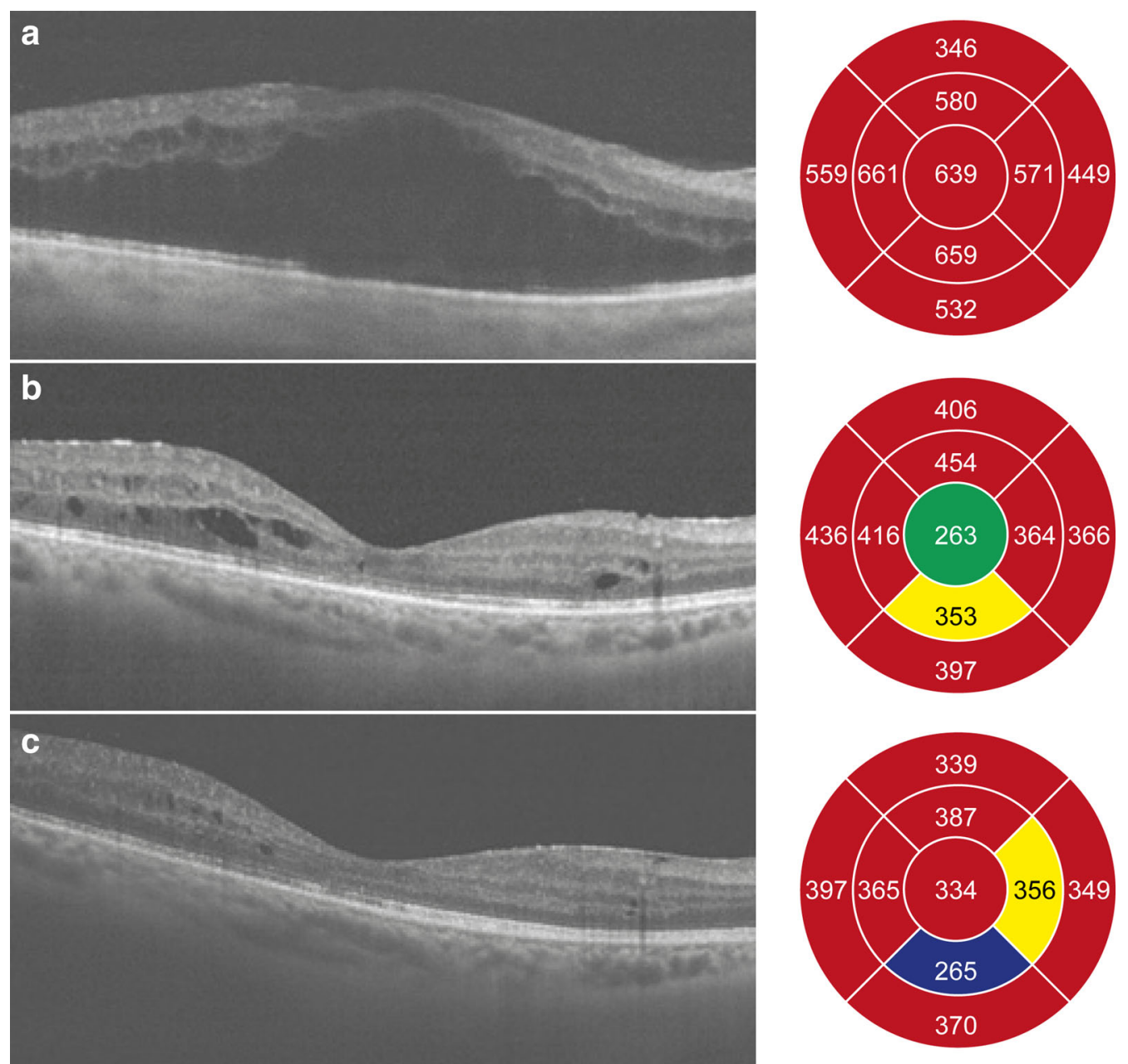

Fig. 4 Changes in macular thickness $(\mu \mathrm{m})$ in the right eye before and after fluocinolone acetonide (FAc) implant. Optical coherence tomography (OCT) scans (left side) and corresponding retinal thickness maps (right side) for right eye diabetic maculopathy in a patient with diabetic macular edema (DME); a before FAc implant but following

three further injections of ranibizumab. In light of the lack of response to anti-VEGF injections and subsequent response to FAc implant in the right eye, it may have been appropriate to consider FAc implant sooner. An empirical observation here is that the fellow eye could be a useful guide to subsequent responses in the contralateral eye. This of course needs to be explored further and will hopefully be answered through additional patient cases or studies. eighteen injections of ranibizumab; $\mathbf{b} 1$ month following FAc implant; c 6 months after FAc implant. Color indications: Green (displays retinal thickness within normal limits), Yellow (retinal thickness marginally outside normal limits), Red (retinal thickness substantially outside normal limits), Blue (shows reduction in macular thickness)

In addition to the measurable clinical benefit, it is vitally important to consider the patient's perspective. In the current case, the patient worked as a window cleaner on high-rise buildings, but was unable to continue working prior to FAc implant. Following the implant, which may have improved patient compliance as a long-lasting (up to 3 years) consistent therapy, he indicated he felt comfortable returning to work and restarted his previous job. This reflects 
an important, real-world functional benefit resulting from the improvement in BCVA to 0.5, or $20 / 40$ in the left eye; an outcome also important as it is the minimum requirement for maintaining a driving license in Europe [11].

In this case, an initial challenge with intraocular steroid was not provided as this is not required in the European SmPC guidelines [8]. However, it should be noted that the United States Food and Drug Administration (FDA) prescribing information does stipulate that patients, 'have been previously treated with a course of corticosteroids and did not have a clinically significant rise in intraocular pressure' [10].

\section{CONCLUSION}

This case report demonstrates the potential effectiveness of ILUVIEN in a real-world clinical setting, and suggests that FAc implant or another corticosteroid should be considered if the patient does not respond optimally to an anti-VEGF and/or laser treatment. FAc implant may provide additional treatment compliance benefits as a result of the sustained low-dose delivery of FAc $(0.2 \mu \mathrm{g} /$ day) for up to 3 years [2]. The current case is the first, that the authors are aware of, to show: (a) a direct comparison between the responses to FAc implant and anti-VEGF injection; and, (b) that when a patient is not responding optimally to antiVEGF therapy, FAc implant was effective and provided similar effects to those achieved in the contralateral eye previously treated with FAc implant.

\section{ACKNOWLEDGMENTS}

Medical writing and editorial assistance were provided by Sarah Jane Rutherford, QXV Communications, Macclesfield, UK and was funded by Alimera Sciences. The research and publication of this article were supported by Alimera Sciences Ltd. All named authors meet ICMJE criteria for authorship for this manuscript, take responsibility for the integrity of the work as a whole, and have given final approval for the version to be published.

Conflict of interest. Dr. Bertelmann and Dr. Schulze have no conflict of interest relating to this publication.

Compliance with ethics. Informed consent was obtained from the patients for being included in the study.

Open Access. This article is distributed under the terms of the Creative Commons Attribution Noncommercial License which permits any noncommercial use, distribution, and reproduction in any medium, provided the original author(s) and the source are credited.

\section{REFERENCES}

1. Virgili G, Parravano M, Menchini F, Evans JR. Antivascular endothelial growth factor for diabetic macular oedema. Cochrane Database Syst Rev. 2014;10:CD007419.

2. Campochiaro PA, Brown DM, Pearson A, et al. Sustained delivery fluocinolone acetonide vitreous inserts provide benefit for at least 3 years in patients with diabetic macular edema. Ophthalmology. 2012;119:2125-32.

3. Messenger WB, Beardsley RM, Flaxel CJ. Fluocinolone acetonide intravitreal implant for the treatment of diabetic macular edema. Drug Des Dev Ther. 2013;7:425-34.

4. Schwartz SG, Flynn HW Jr. Fluocinolone acetonide implantable device for diabetic retinopathy. Curr Pharm Biotechnol. 2011;12:347-51.

5. Brown DM, Nguyen QD, Marcus DM, et al. Longterm outcomes of ranibizumab therapy for diabetic macular edema: the 36-month results from two 
phase III trials: RISE and RIDE. Ophthalmology. 2013;120:2013-22.

6. Campochiaro PA, Nguyen QD, Hafiz G, et al. Aqueous levels of fluocinolone acetonide after administration of fluocinolone acetonide inserts or fluocinolone acetonide implants. Ophthalmology. 2013;120:583-7.

7. Sanford M. Fluocinolone acetonide intravitreal implant (lluvien $^{\circledR}$ ): in diabetic macular oedema. Drugs. 2013;73:187-93.

8. SmPC guidelines. https://www.medicines.org.uk/ emc/medicine/27636. Accessed Nov 24, 2014.

9. Bertelmann T, Messerschmidt-Roth A. Intravitreal ILUVIEN $^{\circledR}$ implant for diabetic macular oedema- early case experiences. Eur Ophthalmic Rev. $2013 ; 7: 122-6$.

10. ILUVIEN $^{\circledR}$ prescribing information. Alimera Sciences, Inc. 6120 Windward Parkway, Alpharetta, GA 30005. http://www.alimerasciences. com/wp-content/uploads/2014/09/iluvien-prescribinginformation.pdf. Accessed Jan 8, 2015.

11. Bron AM, Viswanathan AC, Thelen $\mathrm{U}$, et al. International vision requirements for driver licensing and disability pensions: using a milestone approach in characterization of progressive eye disease. Clin Ophthalmol. 2010;4:1361-9. 